\title{
On Some Spectral Problems for Diffusion Operator
}

\author{
Mine Babaoglu, , Etibar S. Panakhov ${ }^{2}$
}

${ }^{1}$ Kahramanmaras Sutcu Imam University, Faculty of Education, Kahramanmaras, Turkey

${ }^{2}$ Baku State University, Institute of Applied Mathematics, Baku, Azerbaijan

Abstract. In this study, we attain several spectral results for Diffusion operator. In particular, the solution functions belong to Paley-Wiener space:

$P W_{\pi}=\left\{f\right.$ entire, $\left.|f(\mu)| \leq C e^{\pi|\operatorname{Im} \mu|}, \int_{R}|f(\mu)|^{2} d \mu<\infty\right\}$.

so that required theorems are proved.

\section{Introduction}

The aim of this paper is to solve the following diffusion equation

$$
-y^{\prime \prime}+[2 \lambda p(x)+q(x)] y=\lambda^{2} y, \quad x \in[0, \pi]
$$

where the functions $p(x)$ and $q(x)$ are real-valued and $p(x) \in W_{2}^{m+1}[0, \pi]$, $q(x) \in W_{2}^{m}[0, \pi]$ for $m \geq 1$. Some spectral problems were extensively solved for the diffusion operator in references [1-4].

Consider the problem

$$
\begin{aligned}
& -y^{\prime \prime}+[2 \lambda p(x)+q(x)] y=\lambda^{2} y, \\
& y(0)=1, \quad y^{\prime}(0)=h,
\end{aligned}
$$

where $h$ is a finite number. Let us denote by $\varphi(x, \lambda)$ the solution of (2) satisfying the initial conditions (3). Let [2]

$$
\varphi(x, \lambda)=\cos [\lambda x-\alpha(x)]+\int_{0}^{x} A(x, t) \cos \lambda t d t+\int_{0}^{x} B(x, t) \sin \lambda t d t,
$$

where

$$
\alpha(x)=x \cdot p(0)+2 \int_{0}^{x}\{A(\xi, \xi) \sin \alpha(\xi)-B(\xi, \xi) \cos \alpha(\xi)\} d \xi
$$

\footnotetext{
* Corresponding author: mnbabaoglu@gmail.com
} 


$$
\begin{aligned}
& q(x)=-p^{2}(x)+2 \frac{d}{d x}\{A(x, x) \cos \alpha(x)+B(x, x) \sin \alpha(x)\} \\
& A(0,0)=h, \quad B(x, 0)=0,\left.\quad \frac{\partial A(x, t)}{\partial t}\right|_{t=0}=0, \quad \alpha(x)=\int_{0}^{x} p(t) d t
\end{aligned}
$$

and

$$
\lambda_{n}=n+c_{0}+\frac{c_{1}}{n}+\frac{c_{1, n}}{n},
$$

be the $n$th eigenvalue where

$$
\begin{aligned}
& c_{0}=\frac{1}{\pi} \int_{0}^{\pi} p(x) d x, \quad \sum_{n}\left|c_{1, n}\right|^{2}<\infty, \\
& c_{1}=\frac{1}{\pi}\left(h+H+\frac{1}{2} \int_{0}^{\pi}\left[q(x)+p^{2}(x)\right] d x\right),
\end{aligned}
$$

and $H$ is a finite number.

Consider the Diffusion equation with more general separable boundary conditions

$$
\begin{aligned}
& -y^{\prime \prime}+[2 \lambda p(x)+q(x)] y=\lambda^{2} y, \quad x \in[0, \pi], \\
& a_{11} y(0, \lambda)-a_{12} y^{\prime}(0, \lambda)=0, \\
& a_{21} y(\pi, \lambda)+a_{22} y^{\prime}(\pi, \lambda)=0 .
\end{aligned}
$$

where $a_{11}^{2}+a_{12}^{2} \neq 0, a_{21}^{2}+a_{22}^{2} \neq 0$. So, let $\lambda=\mu^{2}$ and $y\left(x, \mu^{2}\right)$ denote the solution of the initial value problem

$$
\begin{gathered}
-y^{\prime \prime}+\left[2 \mu^{2} p(x)+q(x)\right] y=\mu^{4} y, \\
y\left(0, \mu^{2}\right)=a_{12}, \quad y^{\prime}\left(0, \mu^{2}\right)=a_{11} .
\end{gathered}
$$

The eigenvalues of (8) are the square of the zeroes of the boundary function $B(\mu)$,

$$
B(\mu):=a_{21} y\left(\pi, \mu^{2}\right)+a_{22} y^{\prime}\left(\pi, \mu^{2}\right) .
$$

In the Dirichlet case, this boundary function is an entire function of $\mu$ of order 1 and type $\pi$ and is square integrable on the real line. Therefore, it belongs to the Paley-Wiener space.

\section{Main Results}

Let

$$
\begin{aligned}
v_{1}(x, \mu) & =y\left(x, \mu^{2}\right)-\cos \left[\mu^{2} x-\alpha(x)\right] \\
& =\int_{0}^{x} A(x, t) \cos \left(\mu^{2} t\right) d t+\int_{0}^{x} B(x, t) \sin \left(\mu^{2} t\right) d t
\end{aligned}
$$

In the following, we shall make use of the estimates [5],

$$
|\cos u| \leq e^{|\operatorname{Im} u|}, \quad|\sin u| \leq c_{0} e^{|\operatorname{Im} u|},
$$

where $c_{0}$ is some constant (we may take $c_{0}=1.72$ for numerical purposes).

Define the constants 


$$
\begin{aligned}
& c_{1}=\int_{0}^{\pi} \max _{0 \leq x \leq \pi}|A(x, t)| d t, \quad c_{2}=\int_{0}^{\pi} \max _{0 \leq x \leq \pi}|B(x, t)| d t, \\
& c_{3}=\left(c_{1}+c_{0} c_{2}\right)\left|a_{21}\right|+c_{0} c_{2}\left|a_{22}\right| .
\end{aligned}
$$

we claim the subsequent results.

Theorem $1 v_{1}(x, \mu) \in P W_{x}$ is a function of $\mu$ for each $x$ and the following estimate hold:

$$
\left|v_{1}(x, \mu)\right| \leq\left(c_{1}+c_{0} c_{2}\right) e^{x\left|\operatorname{Im} \mu^{2}\right|}
$$

Proof In the first instance

$$
\left|v_{1}(x, \mu)\right| \leq \int_{0}^{x}|A(x, t)|\left|\cos \left(\mu^{2} t\right)\right| d t+\int_{0}^{x}|B(x, t)|\left|\sin \left(\mu^{2} t\right)\right| d t
$$

Thus,

$$
\begin{aligned}
\left|v_{1}(x, \mu)\right| & \leq \int_{0}^{x}|A(x, t)| e^{\left|\operatorname{Im}\left(\mu^{2} t\right)\right|} d t+c_{0} \int_{0}^{x}|B(x, t)| e^{\left|\operatorname{Im}\left(\mu^{2} t\right)\right|} d t \\
& \leq e^{x \mid \operatorname{Im} \mu^{2}} \mid\left(\int_{0}^{x}|A(x, t)| d t+c_{0} \int_{0}^{x}|B(x, t)| d t\right) \\
& \leq e^{x \mid \operatorname{Im} \mu^{2}} \mid\left(\int_{0}^{\pi} \max _{0 \leq x \leq \pi}(|A(x, t)|) d t+c_{0} \int_{0}^{\pi} \max _{0 \leq x \leq \pi}(|B(x, t)|) d t\right)
\end{aligned}
$$

from which we obtain

$$
\left|v_{1}(x, \mu)\right| \leq e^{x\left|\operatorname{Im} \mu^{2}\right|}\left(c_{1}+c_{0} c_{2}\right)
$$

So, we have proved the estimate. Therefore, $v_{1}(x, \mu)$ is entirely of type $x$ order 1 and square integrable on the real line as a function of $\mu$ for each $x$.

Theorem $2 v_{2}(x, \mu) \in P W_{x}$ is a function of $\mu$ for each $x$ and the following estimate hold:

$$
\left|v_{2}(x, \mu)\right| \leq c_{0} c_{2} e^{x\left|\operatorname{Im} \mu^{2}\right|}
$$

\section{Proof}

$$
\begin{aligned}
v_{2}(x, \mu)=\int_{0}^{x}\left\{A(x, t) \cos \left(\mu^{2} t\right) d t+B(x, t) \sin \left(\mu^{2} t\right)\right\} d t-\int_{0}^{x}\left\{A(x, t) \cos \left(\mu^{2} t\right)\right\} d t \\
\left|v_{2}(x, \mu)\right| \leq \int_{0}^{x}|B(x, t)|\left|\sin \left(\mu^{2} t\right)\right| d t \\
\leq \int_{0}^{x} c_{0}|B(x, t)| e^{\left|\operatorname{Im}\left(\mu^{2} t\right)\right|} d t \\
\leq c_{0} e^{x\left|\operatorname{Im} \mu^{2}\right|} \mid\left(\int_{0}^{\pi} \max _{0 \leq x \leq \pi}|B(x, t)| d t\right)
\end{aligned}
$$

from which we get 


$$
\left|v_{2}(x, \mu)\right| \leq\left(c_{0} c_{2}\right) e^{x\left|\operatorname{Im} \mu^{2}\right|}
$$

Hence, function $v_{2}(x, \mu)$ is entirely of type $x$ order 1 and square integrable on the real line as a function of $\mu$ for each $x$.

The boundary function(characteristic equation) $B(\mu)$ is not necessarily in $P W_{\pi}$ as in the Dirichlet-Dirichlet case. However, we have the following theorem.

Theorem $3 \tilde{B}(\pi, \mu)=a_{21} v_{1}(\pi, \mu)+a_{22} v_{2}(\pi, \mu) \in P W_{\pi}$ is a function of $\mu$ and the following estimate holds:

$$
|\tilde{B}(\pi, \mu)| \leq c_{3} e^{\pi\left|\operatorname{Im} \mu^{2}\right|}
$$

Proof Primarily, we have

from which we acquire

$$
|\tilde{B}(x, \mu)| \leq\left|a_{21}\right|\left|v_{1}(x, \mu)\right|+\left|a_{22}\right|\left|v_{2}(x, \mu)\right|
$$

$$
\begin{aligned}
|\tilde{B}(x, \mu)| & \leq\left|a_{21}\right|\left(c_{1}+c_{0} c_{2}\right) e^{x\left|\operatorname{Im} \mu^{2}\right|}+\left|a_{22}\right| c_{0} c_{2} e^{x\left|\operatorname{Im} \mu^{2}\right|} \\
& \leq e^{x\left|\operatorname{Im} \mu^{2}\right|}\left(\left(c_{1}+c_{0} c_{2}\right)\left|a_{21}\right|+c_{0} c_{2}\left|a_{22}\right|\right)
\end{aligned}
$$

so

$$
|\tilde{B}(\pi, \mu)| \leq c_{3} e^{\pi\left|\operatorname{Im} \mu^{2}\right|}
$$

$|\tilde{B}(\pi, \mu)|$ is easily seen from above mentioned inequality. Hence, the following theorem is applicable.

Theorem 4 Let $f \in P W_{\pi}$, then

$$
f(\mu)=\sum_{k=-\infty}^{\infty} f(k) \frac{\sin \pi(\mu-k)}{\pi(\mu-k)},
$$

where the series converges uniformly on compact set of $\mathbb{R}$ and also in $L_{d \mu}^{2}$ [6].

Let $\tilde{B}_{N}(\pi, \mu)$ denote the truncation of $\tilde{B}(\pi, \mu)$

$$
\tilde{B}_{N}(\pi, \mu)=\sum_{k=-N}^{N} \tilde{B}(\pi, k) \frac{\sin \pi(\mu-k)}{\pi(\mu-k)},
$$

and $B_{N}(\pi, \mu)$ the corresponding approximation to $B(\pi, \mu)$.

\section{Conclusion}

In this paper, we examined Diffusion operator and succeeded in performing our approach for Diffusion equation. The approach is based on the well established technique: Shannon's sampling theorem. Thus, we obtained satisfactory results by using the Paley-Wiener spaces. 


\section{References}

1. E. Bairamov, Ö. Çakar, and A. O. Çelebi, Quadratic pencil of Schrödinger operators with spectral singularities, discrete spectrum and principal functions, J. Math. Anal. Appl. 216 (1997), 303-320.

2. M. G. Gasymov, and G. Sh. Guseinov, Determination of diffusion operator on spectral data, Dokl. Akad. Nauk Azerb. SSSR 37, 2 (1981), 19-23.

3. H. Koyunbakan, and E. S. Panakhov, Half inverse problem for diffusion operators on the finite interval, J. Math. Anal. Appl. 326 (2007), 1024-1030.

4. B. Chanane, Sturm-Liouville problems with parameter dependent potential and boundary conditions, Journal of Computational and Applied Mathematics 212 (2008), 282-290.

5. K. Chadan, and P.C. Sabatier, Inverse Problems in Quantum Scattering Theory, Second edition, Springer-Verlag (1989).

6. A. I. Zayed, Advances in Shannon's Sampling Theory, CRC Press, Boca Raton (1993).

7. B. M. Levitan, and I. S. Sargsjan, Introduction to Spectral Theory: Selfadjoint Ordinary Differential Operators, American Mathematical Society, Providence, Rhode Island (1975).

8. B. Chanane, Computing eigenvalues of regular Sturm-Liouville problems, Appl. Math. Lett. 12 (1999), 119-125.

9. A. Boumenir, and B. Chanane, Computing eigenvalues of Sturm-Liouville systems of Bessel type, Proc. Edinburgh Math. Soc. 42 (1999), 257-265.

10. B. Chanane, Computing the eigenvalues of singular Sturm-Liouville problems using the regularized sampling method, Appl. Math. Com. 184 (2007), 972-978.

11. A. Boumenir, and B. Chanane, Eigenvalues of Sturm-Liouville systems using sampling theory, Applicable Analysis 62 (1996), 323-334.

12. B. Chanane, High Order Approximations of the Eigenvalues of Regular SturmLiouville Problems, J. Math. Anal. Appl. 226 (1998), 121-129. 